\title{
miRNA-301a induces apoptosis of chronic myelogenous leukemia cells by directly targeting TIMP2/ERK1/2 and AKT pathways
}

\author{
XIANBAO HUANG, LING QI, WEI LU, GANGPING YANG, YAN CHEN, RONGYAN ZHANG, \\ JIA RAO, DEXIANG JI, RUIBIN HUANG and GUOAN CHEN
}

Department of Hematology, The First Affiliated Hospital of Nanchang University, Nanchang, Jiangxi 330006, P.R. China

Received July 6, 2016; Accepted November 28, 2016

DOI: $10.3892 /$ or.2016.5330

\begin{abstract}
We investigated the biological functions and mechanism of miRNA-301a on apoptosis in chronic myelogenous leukemia (CML). The expression of miRNA-301a in patient with CML cells was higher than the expression of normal patients. Overall survival (OS) of chronic granulocytic leukemia cell patient with low miRNA-301 expression was superior to that of CML patient with high miRNA-301 expression. Moreover, the upregulation of miRNA-301a increased cell proliferation, inhibited apoptosis and caspase-3 and -9 activity of K562 cells. Next, the upregulation of miRNA-301a suppressed Bax/ $\mathrm{Bcl}-2$ rate and TIMP 2 protein expression, increased phosphorylation-ERK1/2 and decreased phosphorylation-AKT protein expression of K562 cells. Furthermore, si-TIMP2 expression enhanced the upregulation of miRNA-301a on the promotion of cell proliferation, inhibition of apoptosis and caspase-3 and -9 activity, suppression of $\mathrm{Bax} / \mathrm{Bcl}-2$ rate, increasing phosphorylation-ERK1/2 and decreasing phosphorylationAKT protein expression of K562 cells. Taken together, our results clearly suggested that miRNA-301a induces apoptosis of CML cells by directly targeting the TIMP2/ERK1/2 and AKT pathways.
\end{abstract}

\section{Introduction}

Chronic myelogenous leukemia (CML) is one of the common types of chronic leukemia in China that occurs in all age groups (1). Though the traditional therapeutic drugs such as hydroxycarbamide and interferon can improve the symptoms in patients within a certain period, they can neither cure this disease, nor block the disease progression (2). An overwhelming majority of patients will enter the acceleration phase or the blastic phase after a period of chronic phase, and

Correspondence to: Professor Guoan Chen, Department of Hematology, The First Affiliated Hospital of Nanchang University, 17 Yongwaizheng Street, Nanchang, Jiangxi 330006, P.R. China E-mail: chenguoannc@163.com

Key words: miRNA-301a, chronic granulocytic leukemia cell, TIMP2, ERK1/2, AKT finally die of complications such as hematopoietic failure or infection. The fusion gene bcr/abl is the genetic and molecular biological feature of $\mathrm{CML}$, and its product $\mathrm{Bcr} / \mathrm{Abl}$ kinase is the key factor of pathogenesis. Since Bcr/Abl kinase possesses strong tyrosine kinase activity, the tyrosine kinase inhibitors (TKIs) such as imatinib serve as the first-line therapeutic drugs for leukemia applied in clinic (3). It is found during treatment that imatinib shows excellent therapeutic effect on the chronic phase of CML; however, resistance occurs in some patients. As demonstrated in research, Bcr/Abl kinase can activate multiple intracellular signaling pathways, for instance, Ras/ MAPK, Jun/STAT5 and PI3K/Akt/mTOR, and thus influence the cell cycle distribution and cell apoptosis, leading to the genesis of CML.

MicroRNA (miRNA) is a type of single stranded noncoding microRNA that is $20 \mathrm{nt}$ in length, and it mainly exerts its posttranscriptional regulatory function through degrading its target gene or inhibiting its translation by complete or incomplete pairing with the target gene 3'-UTR (4). In-depth research on the mechanisms of the production and precise action of miRNA demonstrated that miRNA plays significant roles in the development regulation, differentiation, proliferation, cell apoptosis and metabolism (5). The disordered expression of miRNA may participate in the genesis and development of a tumor, including some hematological tumors. Because miRNA can directly regulate the expression of some oncogenes or tumor suppressor genes after transcription, it can exert functions that are similar to tumor suppressor genes or oncogenes in the body; for instance, miR-424 can induce apoptosis by targeting oncogene BCL-2; while miR-21 negatively regulates the expression of the tumor suppressor gene PTEN and promotes cell proliferation and invasion $(5,6)$.

Matrix metalloproteinase-2 and -9 (MMP-2 and MMP-9) have the important function of hydrolyzing gelatin, type I, IV, V, VII and X collagens as well as elastin (7). Their corresponding tissue inhibitors of metalloproteinase (TIMP) are TIMP-2 and TIMP-1, respectively (8). It has been reported that many solid tumors, such as human breast cancer, endometrial cancer, brain tumor, colorectal cancer and pancreatic cancer, are associated with high gelatinase expression (9). A recent study indicated that TIMP-2 is closely related to the genesis and development of hematological tumor diseases, such as leukemia and myelodysplastic syndrome (10). 
The common biological feature of a tumor is the uncontrolled growth, the major molecular mechanism of which is the inhibited cell malignant proliferation and apoptosis (11). The multiple pathological mechanisms that are involved in the transformation, proliferation and apoptosis of malignant tumors are correlated with the regulation of signaling pathways, among which, RaS/Raf/ERK signal transduction cascade pathway is one of the important pathways. The extracellular signal-regulated kinase (ERK) is a subgroup of the mitogen actived protein kinase (MAPK) family, which can be divided into ERK1 and ERK2 and is generally termed as ERK1/2 (11). ERK1/2 takes part in cell proliferation, differentiation, transformation and apoptosis after being activated by numerous growth factors and cytokines. Recent research suggests that the excessively activated ERK1/2 is related to the genesis of the tumor (11). ERK is associated with the abnormal expression or enhanced activity in multiple tumor tissues as well as tumor cell lines, such as liver cancer, breast cancer, oral squamous cell carcinoma and prostate cancer (12).

Tumor is a common malignancy, however, the pathogenesis is still unclear (13). With the increasing in-depth research on tumors, more and more signaling pathways that play important regulatory effects on the growth and development of organism are proved to be correlated with tumors, among which there are PI3K/Akt signaling pathway, JAK/STAT signaling pathway, MAPK signaling pathway and Wnt/Notch signaling pathway (14). PI3K/Akt signaling pathway is the most extensively studied, it plays an important role in the development of leukemia (15). Numerous studies have demonstrated that EpsS, which participates in EGFR signaling pathway, Rac signaling pathway, PI3K/Akt signaling pathway and mitosis signaling pathway, also plays an important role in the cell cycle and apoptosis (16). The purpose of this study was to investigate the cancer mediation effect of miRNA-301a on apoptosis of CML and its possible mechanism.

\section{Materials and methods}

Ethics statement. Bone marrow and peripheral blood samples were evaluated from 42 CML patients and 8 normal volunteer at Department of Hematology, The First Affiliated Hospital of Nanchang University from 2012, September to 2012, December. All patients and volunteer were obtained from the clinical trials and biomedical ethics special committee of The First Affiliated Hospital of Nanchang University. Every two months, we made a followed-up of the patients.

Quantitative real-time PCR. Total RNA was extracted from CML patients or normal volunteer samples or cell lines using TRIzol reagent (Invitrogen, Carlsbad, CA, USA). Total RNA $(1 \mu \mathrm{g})$ was synthesized into complementary DNA (cDNA) using reverse transcriptase (Epicentre, Madison, WI, USA). A SYBR Green PCR kit (Takara, Dalian, China) was used in quantitative real-time PCR. The primers for miR-301a were: forward, 5'-GGCAGTGCAATAGTATTGT-3' and reverse, 5'-TGGTGTCGTGGAGTCG-3'. The relative miR-301a expression level was determined by using the $2^{-\Delta \Delta C T}$ method.
Cells and reagents. The K562 cells were cultured in RPMI-1640 (Hyclone, Logan, UT, USA) supplemented with 10\% fetal bovine serum (Gibco, Grand Island, NY, USA) in an incubator maintained at $37^{\circ} \mathrm{C}$ in an atmosphere containing $5 \% \mathrm{CO}_{2}$.

Plasmids transfection. miRNA-301a, si-TIMP2 and negative plasmids were purchased from Genechem (Shanghai, China). K562 cells were transfected using Lipofectamine 2000 transfection reagent (Invitrogen).

Cytotoxicity assay. The K562 cells were seeded in 96-well plates at $1 \times 10^{5}$ cells per well, and $20 \mu 13$-(4,5-dimethylthiazol2-yl)-2, 5-diphenyltetrazolium bromide (MTT) solution (5 mg/ $\mathrm{ml}$ ) was added to each well and incubated at $37^{\circ} \mathrm{C}$ for $4 \mathrm{~h}$. After removing the medium, $100 \mu \mathrm{l}$ of DMSO (Sigma) was added to solubilize the crystals and the absorbance was measured using scanning multiwell spectrophotometer (Model 550, Bio-Rad, Hercules, CA, USA) at $450 \mathrm{~nm}$.

Flow cytometric analysis. The K562 cells were seeded in 6 -well plates at $2 \times 10^{6}$ cells per well and washed twice with phosphate-buffered saline (PBS). K562 cells were incubated with Annexin V-FITC and PI (BD Biosciences) for $15 \mathrm{~min}$ in the dark. Apoptosis rate was analyzed using FACScan flow cytometry and CellQuest analysis software (BD Biosciences, Franklin Lakes, NJ, USA).

Western blotting. The K562 cells were seeded in 6-well plates at $2 \times 10^{6}$ cells per well and washed twice with PBS. Cells were lysed using RIPA buffer containing a protease and phosphatase inhibitor mixture (Roche). Total protein was quantified with the BioRad Dc protein assay kit (Bio-Rad, Richmond, CA, USA). Protein was loaded onto $6-10 \%$ sodium and electro-transferred to polyvinylidene difluoride membranes (Millipore, Billerica, MA, USA). Membranes were incubated with the primary antibody anti-Bax, anti-Bcl-2, anti-TIMP2, anti-phosphorylation-ERK1/2, anti-phosphorylation-AKT and GAPDH overnight at $4^{\circ} \mathrm{C}$, followed by incubation with the anti-rabbit secondary antibody for $1 \mathrm{~h}$ at room temperature. Bands were detected by enhanced chemiluminescence (ECL) detection system (Bio-Rad Laboratories).

Statistical analysis. The results are reported as means \pm standard deviation (SD). Student's t-test, one-way analysis of variance (ANOVA) and the Dunnett-test were used to assess statistical significance. Values of $p<0.05$ were considered statistically significant.

\section{Results}

The expression of miRNA-301a in patient with CML. To explore the expression level of miR-301a in CML patients, we performed quantitative real-time PCR on miRNA-301a expression. Interestingly, we found that miR-301a expression level of CML patients were lower than that of the normal group (Fig. 1).

Overall survival (OS) of patient with CML. We followed up the patient with CML and analyzed the OS of patient with CML and miRNA-301a expression. As shown in Fig. 2, OS of CML 


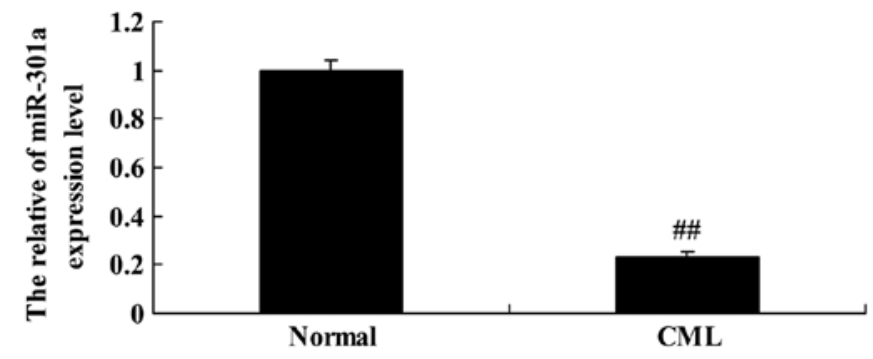

Figure 1. The expression of miRNA-301a in patient with CML. ${ }^{\# \#} \mathrm{p}<0.01$ versus the negative group.

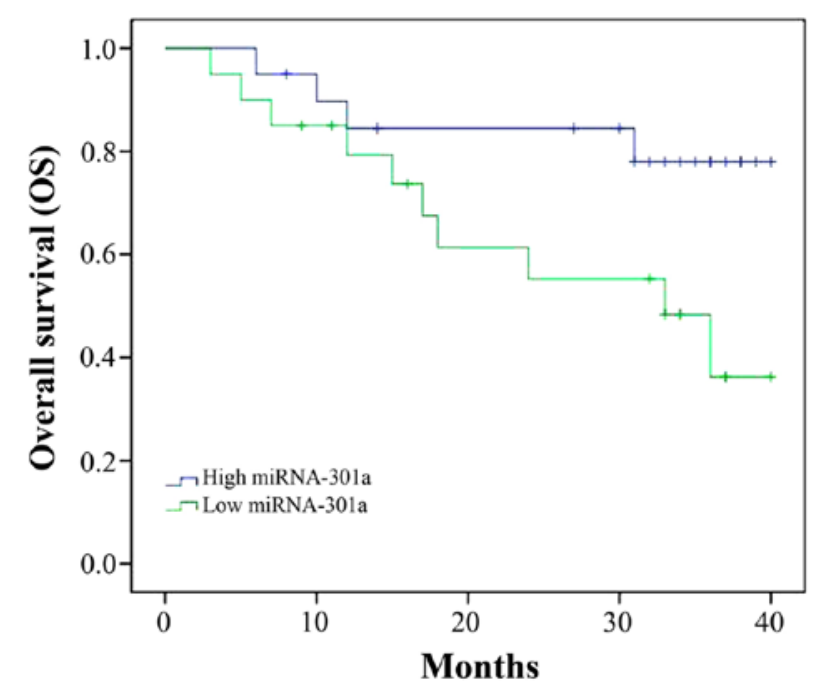

Figure 2. Overall survival (OS) of patient with CML.

patient with high miRNA-301a expression was superior to that of CML patient with low miRNA-301a expression.

Upregulation of miRNA-301a affects cell proliferation of K562 cells. The upregulation of miRNA-301a on cell proliferation of K562 cells was investigated using MTT assay. Fig. 3A shows that miRNA-301a plasmids significantly increased miRNA-301a expression in K562 cells, compared with the negative control group. Then, we found that miRNA-301a upregulation significantly accelerated cell proliferation

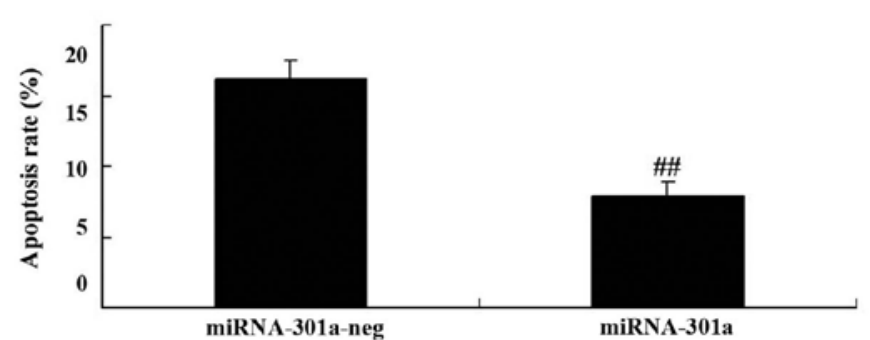

Figure 4. Upregulation of miRNA-301a on apoptosis of K562 cells. miRNA-301a-neg, negative group; miRNA-301a, miRNA-301a group. ${ }^{\# \#} \mathrm{p}<0.01$ versus the negative group.

of K562 cells, compared with the negative control group (Fig. 3B).

Upregulation of miRNA-301a affects apoptosis of K562 cells. To evaluate whether the upregulation of miRNA-301a affect apoptosis of K562 cells, apoptosis was investigated using western blotting. Apoptosis of K562 cells was reduced by upregulation of miRNA-301a expression, compared with negative control group (Fig. 4).

Upregulation of miRNA-301a affects caspase-3 and-9 activity of K562 cells. The effect of miRNA-301a upregulation on caspase-3 and -9 activity of K562 cells, was measured using ELISA kits. As shown in Fig. 5, upregulation of miRNA-301a significantly decreased caspase-3 and -9 activity of K562 cells, compared with negative control group.

Upregulation of miRNA-301a affects Bax/Bcl-2 rate of K562 cells. We observed the upregulation of miRNA-301a on $\mathrm{Bax} / \mathrm{Bcl}-2$ rate of $\mathrm{K} 562$ cells. We found that the upregulation of miRNA-301a significantly suppressed Bax/Bcl-2 rate of K562 cells, compared with negative control group (Fig. 6).

Upregulation of miRNA-301a affects TIMP2 protein expression of $K 562$ cells. To evaluate whether the upregulation of miRNA-301a affects TIMP2 protein expression of K562 cells, western blotting was performed to examine the level of TIMP2 protein of K562 cells with upregulation of miRNA-301a.
A

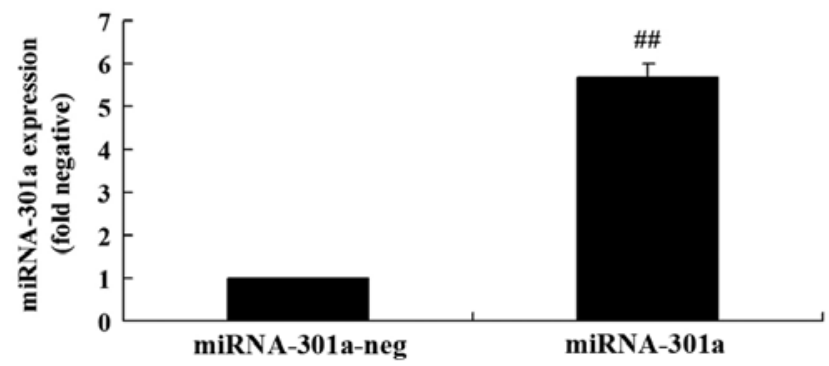

B

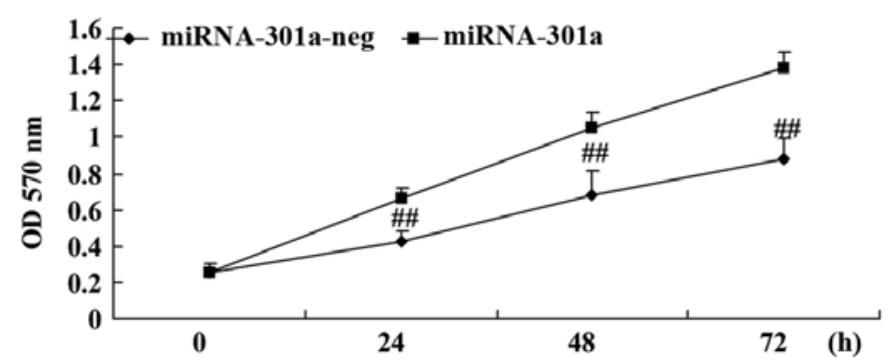

Figure 3. Upregulation of miRNA-301a on cell proliferation of K562 cells. Upregulation of miRNA-301a on miRNA-301a expression (A) and cell proliferation (B) of K562 cells. miRNA-301a-neg, negative group; miRNA-301a, miRNA-301a group. ${ }^{\#}$ p $<0.01$ versus the negative group. 
A

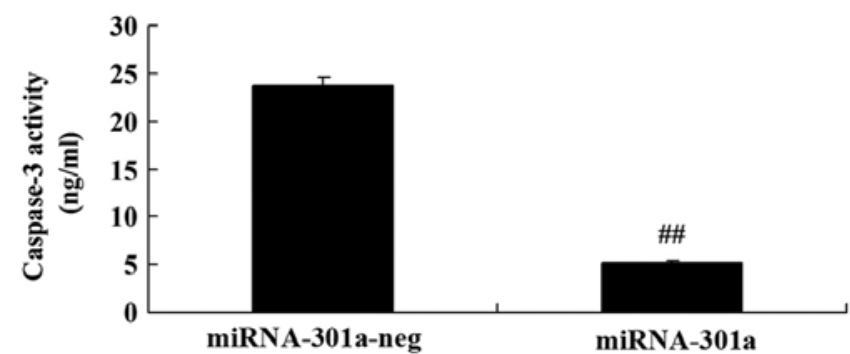

B

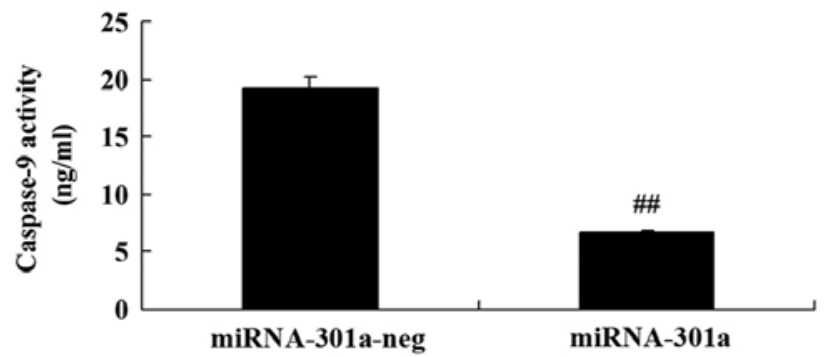

Figure 5. Upregulation of miRNA-301a on caspase-3 and -9 activity of K562 cells. Upregulation of miRNA-301a on caspase-3 (A) and caspase-9 (B) activity of K562 cells. miRNA-301a-neg, negative group; miRNA-301a, miRNA-301a group. ${ }^{\# \#} \mathrm{p}<0.01$ versus the negative group.

A

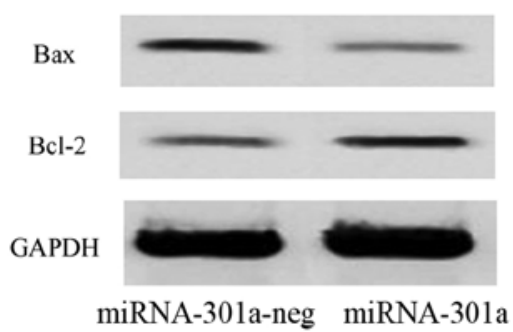

B

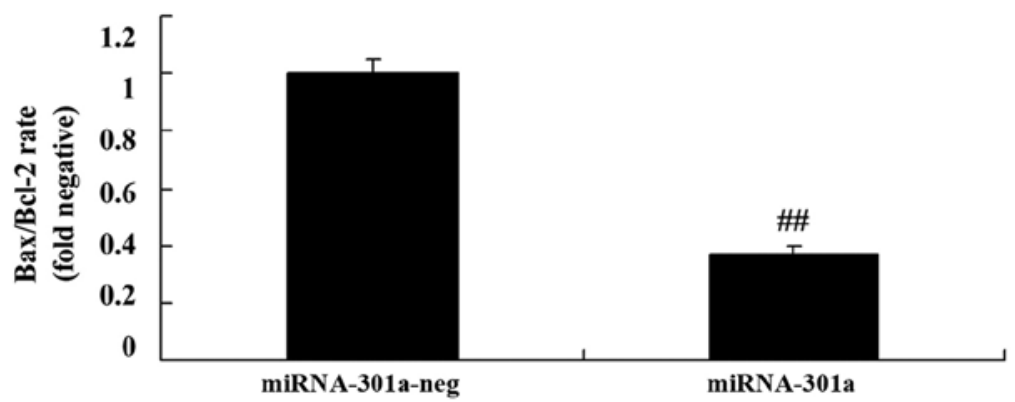

Figure 6. Upregulation of miRNA-301a on Bax/Bcl-2 rate of K562 cells. Upregulation of miRNA-301a on Bax/Bcl-2 protein expression using western blot analysis (A) and statistical analysis (B) of K562 cells. miRNA-301a-neg, negative group; miRNA-301a, miRNA-301a group. ${ }^{\# \#}$ p $<0.01$ versus the negative group.

A

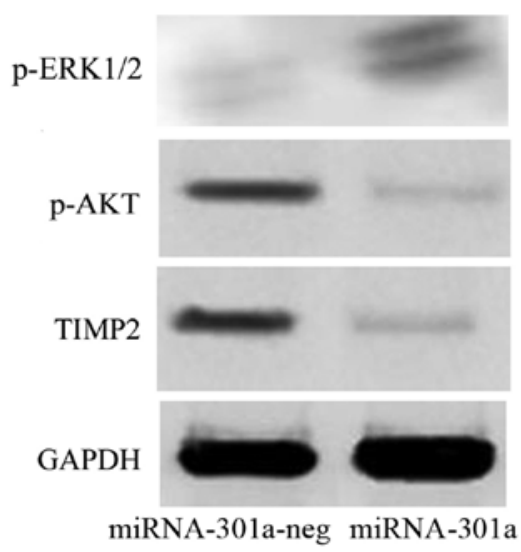

B

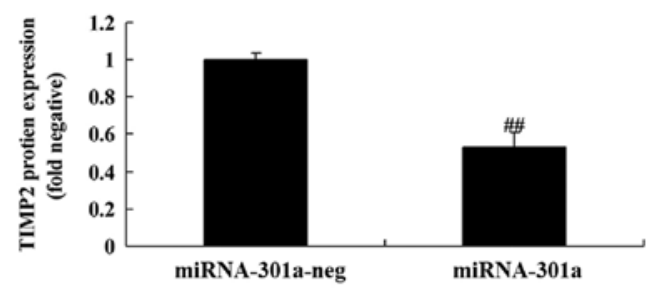

C

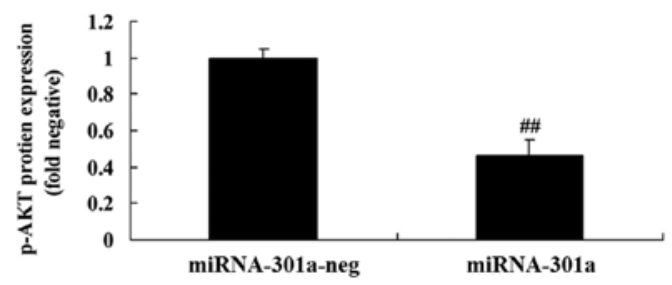

D

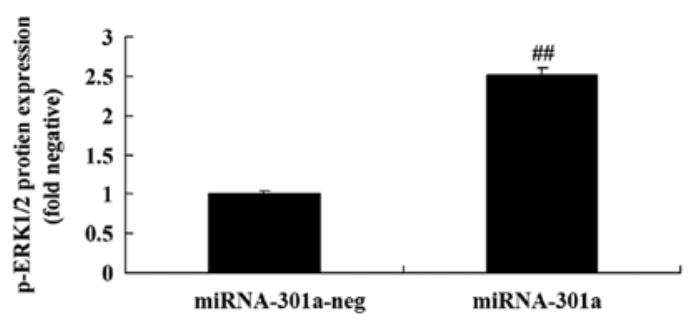

Figure 7. Upregulation of miRNA-301a on TIMP2, Akt and ERK protein expression of K562 cells. Upregulation of miRNA-301a on TIMP2, p-Akt and p-ERK protein expression using western blot analysis (A) and statistical analysis (B-D) of K562 cells. miRNA-301a-neg, negative group; miRNA-301a, miRNA-301a group. ${ }^{\# \#}$ p $<0.01$ versus the negative group. 
A

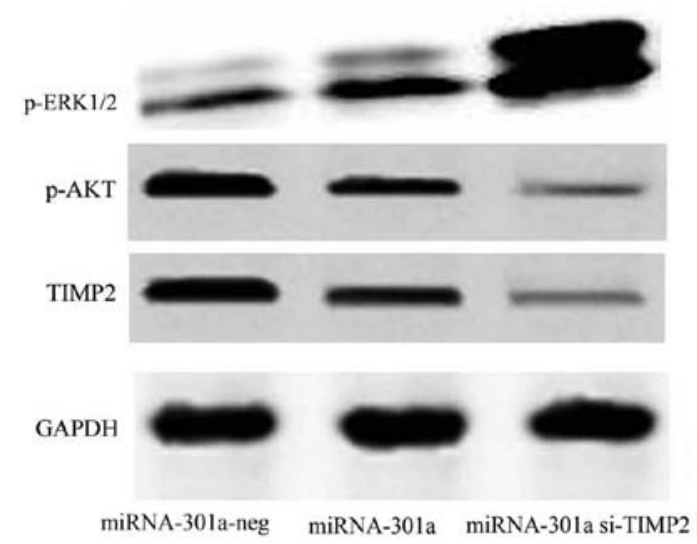

B

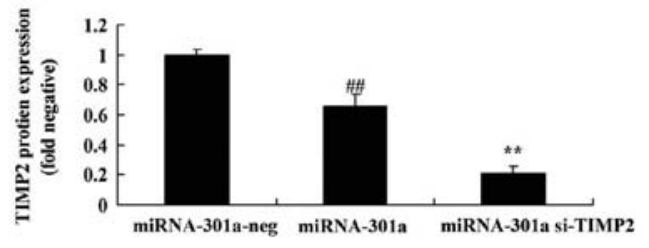

C

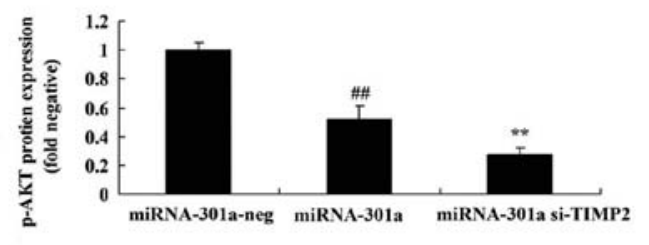

D

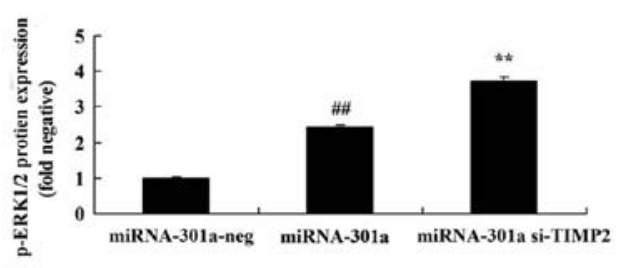

Figure 8. Downregulation of TIMP2 expression influences the effect of miRNA-301a on TIMP2, Akt and ERK protein expression of K562 cells. Downregulation of TIMP2 expression influenced the effect of miRNA-301a on TIMP2, p-Akt and p-ERK protein expression was by western blot analysis (A) and statistical analysis (B-D) of K562 cells. miRNA-301a-neg, negative group; miRNA-301a, miRNA-301a group; miRNA-301a si-TIMP2, miRNA-301a si-TIMP2 group. ${ }^{\# \#} \mathrm{p}<0.01$ versus the negative group. ${ }^{* *} \mathrm{p}<0.01$ versus the miRNA-301a group.

TIMP2 protein expression of K562 cells was significantly decreased by upregulation of miRNA-301a, compared with negative control group (Fig. 7A and B).

Upregulation of miRNA-301a affect on Akt protein expression of $K 562$ cells. Akt is an anti-apoptotic protein, we inspected p-Akt protein expression of K562 cells after miRNA-301a transfection. As shown in Fig. 7A and C, upregulation of miRNA-301a significantly suppressed p-Akt protein expression of K562 cells, compared with negative control group.

Upregulation of miRNA-301a on ERK protein expression of K562 cells. We elucidated whether the upregulation of miRNA-301a affects the mechanism apoptosis of K562 cells, p-ERK protein expression was detected using western blotting. The results of western blotting showed that upregulation of miRNA-301a significantly advanced p-ERK protein expression of K562 cells, compared with negative control group (Fig. 7A and D).

Downregulation of TIMP2 expression influences the effect of miRNA-301a on TIMP2 protein expression of K562 cells. We explored the TIMP2 expression of miRNA-301a on cell growth of CML, and transfected si-TIMP2 into K562 cells. As shown in Fig. 8A and B, si-TIMP2 effectively suppressed the TIMP2 protein expression of K562 cells, compared with upregulation of miRNA-301a expression in K562 cells.
Downregulation of TIMP2 expression influences the effect of miRNA-301a on cell proliferation and cell apoptosis of K562 cells. We further examined downregulation of TIMP2 expression of miRNA-301a on cell proliferation and cell apoptosis of K562 cells. We found that the downregulation of TIMP2 expression effectively increased cell proliferation and inhibited cell apoptosis of K562 cell transfection with upregulation of miRNA-301a, compared with upregulation of miRNA-301a expression in K562 cells (Fig. 9).

Downregulation of TIMP2 expression influences the effect of miRNA-301a on caspase-3 and -9 activity of $K 562$ cells. We transfected K562 cells with si-TIMP2 and determine caspase-3 and -9 activity of K562 cells with upregulation of miRNA-301a. Results from ELISA assay showed that downregulation of TIMP2 expression inhibited caspase- 3 and -9 activity of K562 cells with upregulation of miRNA-301a, compared with upregulation of miRNA-301a expression in K562 cells (Fig. 10).

Downregulation of TIMP2 expression influences the effect of miRNA-301a on Bax/Bcl-2 rate of $K 562$ cells. To determine whether TIMP2 would affect K562 cell apoptosis with upregulation of miRNA-301a, Western blotting was performed to analyze the protein expression of Bax and Bcl-2 as shown in Fig. 11. The protein expression of $\mathrm{Bax} / \mathrm{Bcl}-2$ rate was significantly decreased by downregulation of TIMP2 in K562 cells with upregulation of miRNA-301a, compared with upregulation of miRNA-301a expression in K562 cells. 
A

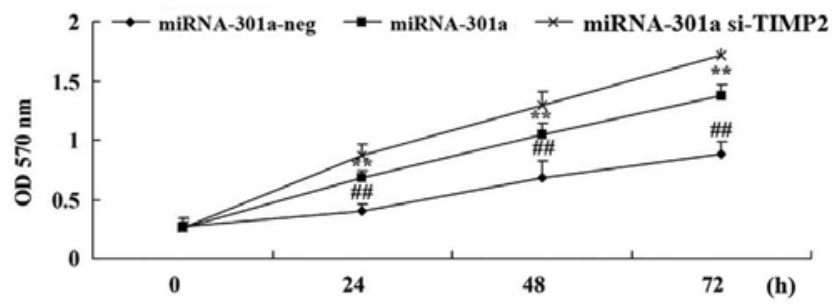

B

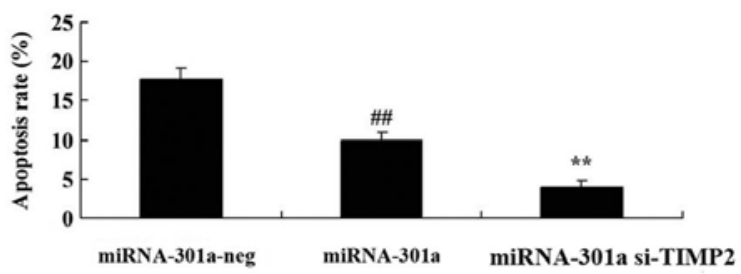

Figure 9. Downregulation of TIMP2 expression influences the effect of miRNA-301a on cell proliferation and apoptosis of K562 cells. Downregulation of TIMP2 expression influenced the effect of miRNA-301a on cell proliferation (A) and apoptosis (B) of K562 cells. miRNA-301a-neg, negative group; miRNA-301a, miRNA-301a group; miRNA-301a si-TIMP2, miRNA-301a si-TIMP2 group. ${ }^{\# \#}$ p $<0.01$ versus the negative group. ${ }^{* *}$ p $<0.01$ versus the miRNA-301a group.

A

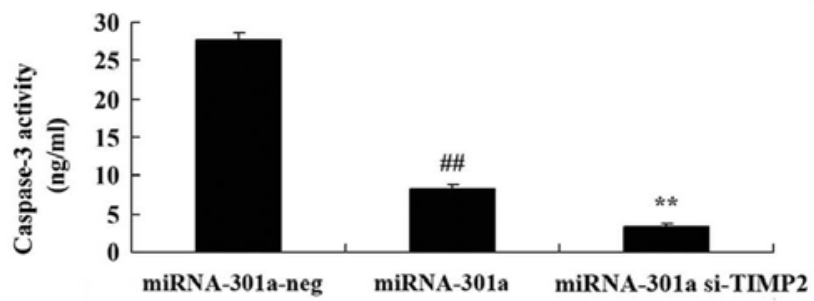

B

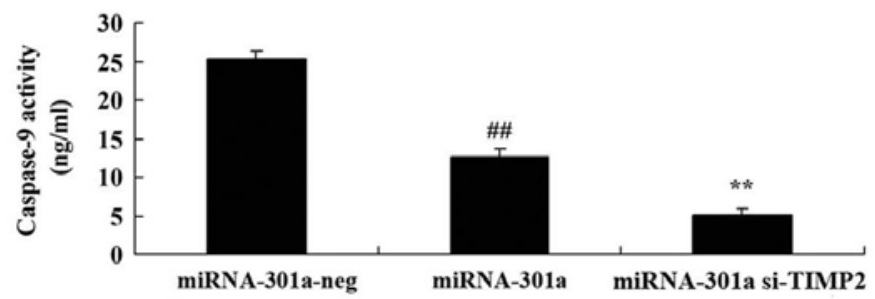

Figure 10. Downregulation of TIMP2 expression influences the effect of miRNA-301a on caspase-3 and -9 activity of K562 cells. Downregulation of TIMP2 expression influenced the effect of miRNA-301a on caspase-3 (A) and caspase-9 (B) activity of K562 cells. miRNA-301a-neg, negative group; miRNA-301a, miRNA-301a group; miRNA-301a si-TIMP2, miRNA-301a si-TIMP2 group. ${ }^{\# \#} \mathrm{p}<0.01$ versus the negative group. ${ }^{* *}$ p $<0.01$ versus the miRNA-301a group.

A

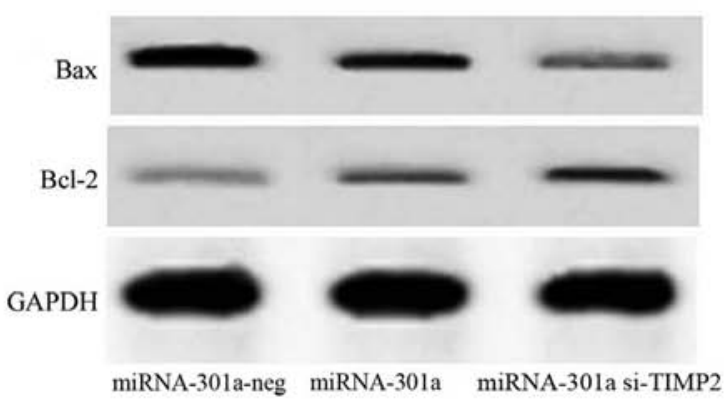

B

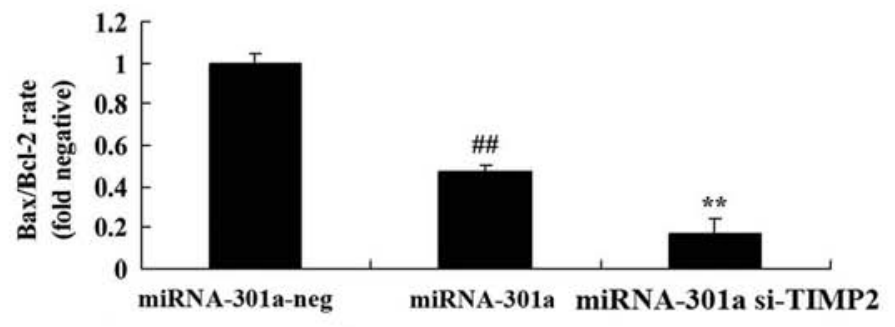

Figure 11. Downregulation of TIMP2 expression influences the effect of miRNA-301a on Bax/Bcl-2 rate of K562 cells. Downregulation of TIMP2 expression influenced the effect of miRNA-301a on Bax/Bcl-2 using western blot analysis (A) and statistical analysis (B) of K562 cells. miRNA-301a-neg, negative

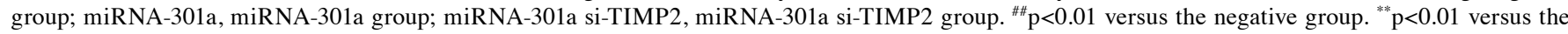
miRNA-301a group.

Downregulation of TIMP2 expression influences the effect of miRNA-301a on Akt protein expression of $K 562$ cells. The TIMP2-mediated signaling was examined to elucidate the Akt activation of K562 cells with upregulation of miRNA-301a. After miRNA-301a transfection, the downregulation of TIMP2 expression significantly suppressed p-Akt protein expression of K562 cells with upregulation of miRNA-301a, compared with upregulation of miRNA-301a expression in K562 cells (Fig. 8A and C).

Downregulation of TIMP2 expression influences the effect of miRNA-301a on ERK protein expression of $\mathrm{K} 562$ cells. We further elucidate whether downregulation of TIMP2 expression influenced the effect of miRNA-301a on ERK protein expression of K562 cells. As shown in Fig. 8A and D, after
miRNA-301a transfection, the downregulation of TIMP2 expression significantly enhanced p-ERK protein expression of K562 cells with upregulation of miRNA-301a, compared with upregulation of miRNA-301a expression in K562 cells.

\section{Discussion}

CML is a common hematological malignant tumor, the morbidity of which accounts for $\sim 20 \%$ among all types of leukemia, with an annual rate of 10/1,000,000 people affected worldwide (17). $\mathrm{Ph}$ chromosome can be detected in CML patients, and the resulting bcr-abl fusion gene encoded P210 Bcr-Abl protein possesses strong tyrosine kinase activity, which results in the phosphorylation of the protein itself, as well as many important substrate molecule tyrosine residues, 
influencing multiple intracellular signal transduction pathways such as Ras/MAPK, PI3K/Akt and JAK/STAT, inducing the malignant transformation of the hematopoietic cells, enhancing the ability of cells to repair DNA injury, inhibiting apoptosis, and mediating resistance of CML to multiple cytotoxic antitumor drugs $(18,19)$. We concluded that miR-301a expression level of CML patients were lower than that of normal group. Yue et al revealed that miR-301a promoted invasion of glioma cells through Wnt/ $\beta$-catenin pathway (20).

The genesis and development of leukemia is an extremely complicated process, which involves genetic and epigenetic changes (4). Upregulated or downregulated expression of miRNA can be detected in various leukemia types, such as CML, chronic lymphocytic leukemia, CML and acute myeloid leukemia (AML) (21). In CML, the changes in miRNA expression render changes of the living environment of the tumor cells, thus accelerating malignant transformation such as tumor cell proliferation, apoptosis escaping, angiogenesis and invasion $(21,22)$. Our results demonstrated that the upregulation of miRNA-301a increased cell proliferation, inhibited apoptosis, caspase-3 and -9 activity, and suppressed Bax/Bcl-2 rate of K562 cells.

MMP-2 exists in the form of zymogen, and the ternary theory is widely accepted at present in terms of its activation, which means that the MMP-14 on the cell membrane binds with TIMP-2 to form the compound, and then TIMP-2 will bind with MMP-2 to form the ternary compound, which renders the easier junction between MMP-2 and another adjacent active MMP-14 in space; moreover, MMP-2 is also activated, thus forming the active form (2). The results demonstrate that there is no expression of MMP-2 and MMP-14 in the normal adult bone marrow mononuclear cells, but someexpression of TIMP-2; all the bone marrow mononuclear cells in CML patients express TIMP-2, and 90\% express MMP-2 and MMP-14 (23). Considering that the chronic phase in CML patients is under the self-balanced status relative to the acute leukemia, while TIMP-2 may be an influencing factor for inhibiting disease progression, the loss of balance may result in the disability to inhibit MMP-2, the excessive expression leads to disease progression (24). Here, we showed that upregulation of miRNA-301a significantly decreased TIMP2 protein expression of K562 cells. Liang et al reported that miR-301a promotes cell proliferation by directly targeting TIMP2 in multiple myeloma (25). These findings suggest that miRNA-301a may function as a novel oncogene in CML contributing to tumor progression of CML.

$\mathrm{P} 210 \mathrm{Bcr}-\mathrm{Abl}$ fusion protein induces the phosphorylation of the tyrosine in multiple intracellular signaling molecules through its high tyrosine kinase activity, thus activating multiple signaling pathways such as Ras/MAPK, PI3K/Akt and JAK/STAT, the activation of these signaling molecules is the molecular basis of the anti-apoptosis of Bcr-Abl (26). The signaling pathway activation downstream of Bcr-Abl also participates in the formation of the resistance to imatinib. CRKL protein is the adaptor protein during the intracellular signal transduction process, which participates in signal transduction pathways like Ras/MAPK JAK/STAT PI3K as well as $\beta 1$ integrin-mediated signal transduction pathway (27). CRKL gene is abundantly expressed in hematopoietic cells, while in normal hematopoietic cells, CRKL protein exists in the form of non-phosphorylated tyrosine. PI3K/Akt and MAPK/ERK are the two important pathways downstream of Bcr/Abl (28). The chronic phase and blastic phase of CML as well as the CML-derived K562 cell lines are associated with the constitutive activation of the ERK and Akt kinase (29). The application of imatinib in K562 cells can inhibit the activity of the ERK and Akt kinase, and induce cell apoptosis through activating caspase-3. However, in some primary leukemia cells in the CML blastic phase, inhibiting the activity of the ERK and Akt kinase can induce caspase-3 mediated apoptosis, giving rise to the occurrence of resistance (30). In this study, we observed that upregulation of miRNA-301a significantly increased phosphorylation-ERK1/2 and decreased phosphorylationAKT protein expression of K562 cell. These results indicate that ERK1/2 and AKT pathways affected miRNA-301a in CML.

Taken together, we demonstrated for the first time that miRNA-301a is significantly upregulated in CML. Moreover, miRNA-301a promotes cell proliferation and inhibits apoptosis of CML by direct targeting TIMP2. These results suggest that miRNA-301a may play as an oncogene in CML and might provide helpful therapeutic strategies for CML in clinical application.

\section{References}

1. Zhou L, Shi H, Jiang S, Ruan C and Liu H: Deep molecular response by IFN- $\alpha$ and dasatinib combination in a patient with T315I-mutated chronic myeloid leukemia. Pharmacogenomics 17: 1159-1163, 2016.

2. Chaudhary AK, Chaudhary S, Ghosh K, Shanmukaiah C and Nadkarni AH: Secretion and expression of matrix metalloproteinase- 2 and 9 from bone marrow mononuclear cells in myelodysplastic syndrome and acute myeloid leukemia. Asian Pac J Cancer Prev 17: 1519-1529, 2016.

3. Janowska-Wieczorek A, Majka M, Marquez-Curtis L, Wertheim JA, Turner AR and Ratajczak MZ: Bcr-abl-positive cells secrete angiogenic factors including matrix metalloproteinases and stimulate angiogenesis in vivo in Matrigel implants. Leukemia 16: 1160-1166, 2002.

4. Di Stefano C, Mirone G, Perna S and Marfe G: The roles of microRNAs in the pathogenesis and drug resistance of chronic myelogenous leukemia (Review). Oncol Rep 35: 614-624, 2016.

5. Hershkovitz-Rokah O, Modai S, Pasmanik-Chor M, Toren A Shomron N, Raanani P, Shpilberg O and Granot G: Restoration of miR-424 suppresses BCR-ABL activity and sensitizes CML cells to imatinib treatment. Cancer Lett 360: 245-256, 2015.

6. Wang WZ, Pu QH, Lin XH, Liu MY, Wu LR, Wu QQ, Chen YH, Liao FF, Zhu JY and Jin XB: Silencing of miR-21 sensitizes CML CD $34^{+}$stem/progenitor cells to imatinib-induced apoptosis by blocking PI3K/AKT pathway. Leuk Res 39: 1117-1124, 2015.

7. Kreisel SH, Stroick M, Griebe M, Alonso A, Reuter B Hennerici MG and Fatar M: True effects or bias? MMP-2 and MMP-9 serum concentrations after acute stroke. Cerebrovasc Dis 42: 352-360, 2016

8. Wu YJ, Neoh CA, Tsao CY, Su JH and Li HH: Sinulariolide suppresses human hepatocellular carcinoma cell migration and invasion by inhibiting matrix metalloproteinase-2/-9 through MAPKs and PI3K/Akt signaling pathways. Int J Mol Sci 16: 16469-16482, 2015.

9. Ries C, Loher F, Zang C, Ismair MG and Petrides PE: Matrix metalloproteinase production by bone marrow mononuclear cells from normal individuals and patients with acute and chronic myeloid leukemia or myelodysplastic syndromes. Clin Cancer Res 5: 1115-1124, 1999.

10. Golubnitschaja O, Yeghiazaryan K, Stricker H, Trog D, Schild HH and Berliner L: Patients with hepatic breast cancer metastases demonstrate highly specific profiles of matrix metalloproteinases MMP-2 and MMP-9 after SIRT treatment as compared to other primary and secondary liver tumours. BMC Cancer 16: 357, 2016. 
11. Chen CC, Liu TY, Huang SP, Ho CT and Huang TC: Differentiation and apoptosis induction by lovastatin and $\gamma$-tocotrienol in HL-60 cells via Ras/ERK/NF- $\kappa \mathrm{B}$ and Ras/Akt/ $\mathrm{NF}-\kappa \mathrm{B}$ signaling dependent down-regulation of glyoxalase 1 and HMG-CoA reductase. Cell Signal 27: 2182-2190, 2015.

12. Liu J, Wang Y, Liu RH and He X: Novel triterpenoids isolated from raisins exert potent antiproliferative activities by targeting mitochondrial and Ras/Raf/ERK signaling in human breast cancer cells. Food Funct 7: 3244-3251, 2016

13. Chien CM, Lin KL, Su JC, Chuang PW, Tseng CH, Chen YL, Chang LS and Lin SR: Naphtho[1,2-b]furan-4,5-dione induces apoptosis of oral squamous cell carcinoma: Involvement of EGF receptor/PI3K/Akt signaling pathway. Eur J Pharmacol 636 : 52-58, 2010.

14. Li Q, Huai L, Zhang C, Wang C, Jia Y, Chen Y, Yu P, Wang H, Rao Q, Wang M, et al: Icaritin induces AML cell apoptosis via the MAPK/ERK and PI3K/AKT signaling pathways. Int J Hematol 97: 617-623, 2013

15. Zeng D, Wang J, Kong P, Chang C, Li J and Li J: Ginsenoside Rg3 inhibits HIF-1 $\alpha$ and VEGF expression in patient with acute leukemia via inhibiting the activation of PI3K/Akt and ERK1/2 pathways. Int J Clin Exp Pathol 7: 2172-2178, 2014.

16. Yi YW, Hong W, Kang HJ, Kim HJ, Zhao W, Wang A, Seong YS and Bae I: Inhibition of the PI3K/AKT pathway potentiates cytotoxicity of EGFR kinase inhibitors in triple-negative breast cancer cells. J Cell Mol Med 17: 648-656, 2013.

17. Becker H, Suciu S, Rüter BH, Platzbecker U, Giagounidis A, Selleslag D, Labar B, Germing U, Salih HR, Muus P, et al: Decitabine versus best supportive care in older patients with refractory anemia with excess blasts in transformation (RAEBt) - results of a subgroup analysis of the randomized phase III study 06011 of the EORTC Leukemia Cooperative Group and German MDS Study Group (GMDSSG). Ann Hematol 94: 2003-2013, 2015.

18. Sakurai M, Mori T, Karigane D, Tozawa K, Matsuki E, Shimizu T, Yokoyama K, Nakajima H, Kanda Y and Okamoto S: Unfavorable outcome of chronic myelogenous leukemia in adolescent and young adults treated with tyrosine kinase inhibitors. Int J Hematol 102: 342-348, 2015.

19. Iriyama $N$, Fujisawa $S$, Yoshida $C$, Wakita $H$, Chiba $S$, Okamoto $S$, Kawakami K, Takezako N, Kumagai T, Inokuchi K, et al: Shorter halving time of BCR-ABL1 transcripts is a novel predictor for achievement of molecular responses in newly diagnosed chronicphase chronic myeloid leukemia treated with dasatinib: Results of the D-first study of Kanto CML study group. Am J Hematol 90 282-287, 2015.

20. Yue X, Cao D, Lan F, Pan Q, Xia T and Yu H: MiR-301a is activated by the Wnt/ $\beta$-catenin pathway and promotes glioma cell invasion by suppressing SEPT7. Neuro-oncol 18: 1288-1296, 2016.
21. Bhutra S,Lenkala D, LaCroix B, Ye M and Huang RS: Identifying and validating a combined mRNA and microRNA signature in response to imatinib treatment in a chronic myeloid leukemia cell line. PLoS One 9: e115003, 2014.

22. Valleron W, Laprevotte E, Gautier EF, Quelen C, Demur C, Delabesse E, Agirre X, Prósper F, Kiss T and Brousset P: Specific small nucleolar RNA expression profiles in acute leukemia. Leukemia 26: 2052-2060, 2012.

23. Song JH, Kim SH, Cho D, Lee IK, Kim HJ and Kim TS: Enhanced invasiveness of drug-resistant acute myeloid leukemia cells through increased expression of matrix metalloproteinase- 2 . Int J Cancer 125: 1074-1081, 2009.

24. Aref S, Osman E, Mansy S, Omer N, Azmy E, Goda T and El-Sherbiny M: Prognostic relevance of circulating matrix metalloproteinase-2 in acute myeloid leukaemia patients. Hematol Oncol 25: 121-126, 2007.

25. Liang B, Yin JJ and Zhan XR: MiR-301a promotes cell proliferation by directly targeting TIMP2 in multiple myeloma. Int J Clin Exp Pathol 8: 9168-9174, 2015.

26. Wang H, Jia XH, Chen JR, Wang JY and Li YJ: Osthole shows the potential to overcome P-glycoprotein-mediated multidrug resistance in human myelogenous leukemia K562/ADM cells by inhibiting the PI3K/Akt signaling pathway. Oncol Rep 35: 3659-3668, 2016

27. Zhang X, Dong W, Zhou H, Li H, Wang N, Miao X and Jia L: $\alpha$-2,8-sialyltransferase is involved in the development of multidrug resistance via PI3K/Akt pathway in human chronic myeloid leukemia. IUBMB Life 67: 77-87, 2015.

28. Rahmani M, Aust MM, Attkisson E, Williams DC Jr, FerreiraGonzalez A and Grant S: Dual inhibition of Bcl-2 and Bcl-xL strikingly enhances PI3K inhibition-induced apoptosis in human myeloid leukemia cells through a GSK3- and Bim-dependent mechanism. Cancer Res 73: 1340-1351, 2013.

29. Chen C, Chang YC, Lan MS and Breslin M: Leptin stimulates ovarian cancer cell growth and inhibits apoptosis by increasing cyclin D1 and Mcl-1 expression via the activation of the MEK/ ERK1/2 and PI3K/Akt signaling pathways. Int J Oncol 42: 1113-1119, 2013.

30. Guo Y, Li Y, Shan Q, He G, Lin J and Gong Y: Curcumin potentiates the anti-leukemia effects of imatinib by downregulation of the AKT/mTOR pathway and BCR/ABL gene expression in $\mathrm{Ph}^{+}$ acute lymphoblastic leukemia. Int J Biochem Cell Biol 65: 1-11, 2015. 